INPLASY

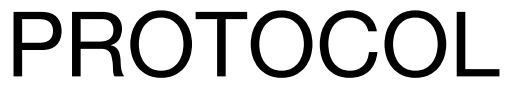

To cite: Zhang et al. Protocol for a systematic review and meta-analysis of Qilong capsule for patients with ischemic stroke. Inplasy protocol 202090057. doi: 10.37766/inplasy2020.9.0057

Received: 13 September 2020

Published: 13 September 2020

Corresponding author:

Cheng Zhang

zc13309604509@163.com

Author Affiliation:

Institute of Basic Research in Clinical Medicine, China

Academy of Chinese

Medical Sciences

Support: 2018YFC1707400.

Review Stage at time of this submission: Preliminary

searches.

Conflicts of interest:

The author(s) declare(s) that there is no conflict of interest regarding the publication of this paper.

\section{Protocol for a systematic review and meta-analysis of Qilong capsule for patients with ischemic stroke}

\author{
Zhang, C1; Geng, HJ2; Zhang, LD³;Xie, YM4.
}

Review question / Objective: Whether Qilong capsule can effectively treat ischemic stroke compared to conventional intervention regimes or western medicine alone?

Condition being studied: Stroke is a common cerebrovascular disease in the world, which is characterized by high incidence, high disability rate, high mortality rate and high recurrence rate. According to the statistical analysis of the global disease burden from 1990 to 2016, stroke was the second leading cause of death in the world in 2016, with 80 million cases of stroke worldwide, of which ischemic stroke accounted for the majority $(84.4 \%)$. Qilong capsule has the effect of replenishing qi and activating blood circulation, removing blood stasis and dredging collaterals, and is used for the treatment of ischemic stroke (TCM syndrome differentiation is qi deficiency and blood stasis syndrome). Studies have confirmed that Qilong capsule can improve the neurological impairment of qi deficiency and blood stasis syndrome in acute ischemic stroke, reduce the content of Pselectin and inhibit platelet activation. At present, there is no systematic study on the efficacy and safety of Qilong capsule in the treatment of ischemic stroke, so it is necessary to conduct a systematic evaluation and meta analysis to provide evidence-based evidence for Qilong capsule in the treatment of ischemic stroke.

INPLASY registration number: This protocol was registered with the International Platform of Registered Systematic Review and Meta-Analysis Protocols (INPLASY) on 13 September 2020 and was last updated on 13 September 2020 (registration number INPLASY202090057).

\section{INTRODUCTION}

Review question / Objective: Whether Qilong capsule can effectively treat ischemic stroke compared to conventional intervention regimes or western medicine alone? 
Condition being studied: Stroke is a common cerebrovascular disease in the world, which is characterized by high incidence, high disability rate, high mortality rate and high recurrence rate. According to the statistical analysis of the global disease burden from 1990 to 2016, stroke was the second leading cause of death in the world in 2016, with 80 million cases of stroke worldwide, of which ischemic stroke accounted for the majority $(84.4 \%)$. Qilong capsule has the effect of replenishing qi and activating blood circulation, removing blood stasis and dredging collaterals, and is used for the treatment of ischemic stroke (TCM syndrome differentiation is qi deficiency and blood stasis syndrome). Studies have confirmed that Qilong capsule can improve the neurological impairment of qi deficiency and blood stasis syndrome in acute ischemic stroke, reduce the content of P-selectin and inhibit platelet activation. At present, there is no systematic study on the efficacy and safety of Qilong capsule in the treatment of ischemic stroke, so it is necessary to conduct a systematic evaluation and meta analysis to provide evidence-based evidence for Qilong capsule in the treatment of ischemic stroke.

\section{METHODS}

Participant or population: The patients will be adults (18 years old or more) who were diagnosed as ischemic stroke. No limitations on country, ethnicity, gender, economic status and educational background will be implemented.

Intervention: Qilong capsule alone or combined with Qilong capsule on the basis of intervention in the control group.

Comparator: Use conventional treatment alone or western medicine.

Study designs to be included: The published or unpublished randomized controlled trials (RCT) and non-randomized controlled trials were included, which explored the efficacy and safety of Qilong capsule in the treatment of ischemic stroke.

Eligibility criteria: Including RCT (excluding quasi-RCT and cluster RCT) and nonrandomized clinical trials. Studies on animal mechanisms will be excluded. Articles that substantially overlap with another article published in print or electronic media will be excluded. Duplicate publications produced by a single experiment will be published as separate papers based on different criteria for measuring the results, and priority will be given to original publications, while other publications will not be included. There is no limit to the language and time of publication.

Information sources: We will manually search electronic databases including PubMed, Embase, Cochrane Library, Web of Science, CNKI, Wanfang, VIP and CBM. The search includes grey literature from CCPD (China Conference papers Database).

Main outcome(s): Primary outcomes are the proportion of recurrent ischemic stroke, symptomatic intracerebral haemorrhage, and the NIHSS score.

Additional outcome(s): functional improvement, as measured by the validated Barthel index or other scales; quality of life, as assessed by the 36-Item Short Form Health Survey; and frequency and severity of adverse events.

Quality assessment / Risk of bias analysis: All the included studies will be evaluated based on the guidelines of Cochrane Handbook for Systematic Reviews of Interventions. The quality of each trial will be categorized into 'low', 'unclear', or 'high' risk of bias according to the following items: adequacy of generation of the allocation sequence, allocation concealment, blinding of participants and personal, blinding of outcome assessors, incomplete outcome data, selected reporting the results and other sources of bias (such as comparable baseline 
characteristic, inclusion and exclusion criteria.

Strategy of data synthesis: We used Revman 5.3 software provided by the Cochrane collaboration to analyze the data. Binary outcomes will be summarized using risk ratio (RR) with $95 \%$ confidence interval (CI) for relative effect. Continuous outcomes will be summarized by using weighted mean difference (WMD) with $95 \%$ CI. We will use random-effect model (REM) for meta-analysis in this review according to research recommendations. Statistical heterogeneity will be assessed by $X^{2}$ and 12 statistical tests. Where $p$ value $\geq 0.1$ and $I 2$ $\leq 50 \%$, there is no obvious statistical heterogeneity among the studies. On the contrary, where $\mathrm{p}$ value $50 \%$ indicates a considerable heterogeneity. Meta-analysis will be performed when the statistical heterogeneity is acceptable ( $p$ value $\geq 0.1$ and $12 \leq 50 \%$ ), otherwise, subgroup analysis will be applied to explore.

Subgroup analysis: We will carry on the subgroup analysis according to the course of treatment and dose. If meta-analysis cannot be carried out, we will conduct a descriptive analysis.

Sensibility analysis: We will conduct a sensitivity analysis to determine the reliability and stability of the summary results by eliminating the tests with high bias risk.

Language: Chinese.

Country(ies) involved: China.

Keywords: Systematic review; Qilong capsule; ischemic stroke.

Contributions of each author:

Author 1 - Cheng Zhang - Author 1 will draft the manuscript.

Author 2 - Hongjiao Geng - The author will contribute to the development of the selection criteria, and the risk of bias assessment strategy.

Author 3 - Lidan Zhang - The author will contribute to the development of the selection criteria, and the risk of bias assessment strategy.

Author 4 - Yanming Xie - The author will read, provide feedback and approve the final manuscript. 